\title{
Distal Protection in Native Coronary Arteries During Primary Angioplasty in Acute Myocardial Infarction: Single-Center Experience
}

\author{
Pedro Silva Orrego, ${ }^{1 *}$ MD, Anabella Delgado, ${ }^{1}$ MD, Giacomo Piccalò, ${ }^{1}$ Paolo Salvadè, ${ }^{1}$ \\ Edgardo Bonacina, ${ }^{2} \mathrm{MD}$, and Silvio Klugmann, ${ }^{1} \mathrm{MD}$
}

\begin{abstract}
Distal embolization of plaque or thrombotic debris is one of the mechanisms involved in the ischemia/reperfusion injury during primary percutaneous intervention for acute occlusion of a native coronary artery. We tested the clinical application of maximal antiplatelet therapy with abciximab combined with one of two different systems of mechanical distal protection: balloon occlusion and aspiration (PercuSurge) in 24 cases and a distal filter (FilterWire Ex) in $\mathbf{1 0}$ cases. Feasibility, technical limitations, and pitfalls are described. Catheter Cardiovasc Interv 2003;60:152-158. @ 2003 Wiley-Liss, Inc.
\end{abstract}

Key words: angioplasty; acute myocardial infarction; embolization

\section{INTRODUCTION}

It is now clear that mechanical reperfusion, when feasible, is superior to systemic thrombolysis in patients with acute myocardial infarction (AMI) [1]. In selected patients, randomized trials have demonstrated that reperfusion with percutaneous coronary interventions (PCIs) compared with intravenous thrombolysis has greater myocardial salvage [2] with better Thrombolysis in Myocardial Infarction (TIMI) flow grade, less mortality, and recurrence of ischemia [3]. Recent studies also suggests that better short- and long-term results with PCI in acute myocardial infarction are obtained with stenting and abciximab [2-5].

The presence of a large thrombus load at the lesion site, however, may provide an increased risk of distal embolization of thrombus or plaque material during balloon inflation or stent deployment [6-11], with deterioration or absence of distal flow and further infarct extension. Angiographic evidence of distal embolization in patients treated with primary angioplasty occurs in approximately $15 \%$ of cases and is associated with a more extensive myocardial damage and a poor prognosis than when distal embolization is not seen [12].

Mechanical distal protection in other situations at risk of embolization, for example saphenous vein grafts (SVGs), has demonstrated clinical benefit with less embolization, better post-PCI coronary flow, and less nonQ-wave myocardial infarction [13,14]. Little is known about entrapment devices in native coronary arteries to prevent embolic damage in the setting of acute myocardial infarction. We have previously described our initial experience with distal protection during PCI in patients with acute myocardial infarction [15]. Here we report the clinical and angiographic findings of PCI with two different systems of distal protection in native coronary arteries during acute myocardial infarction

\section{MATERIALS AND METHODS}

\section{Patient Population}

Thirty-four patients undergoing primary PCI for acute myocardial infarction within $12 \mathrm{hr}$ from symptom onset were treated with one of two mechanical distal protection (MDP) systems. The first 24 patients were treated with a distal balloon and occlusion system (PercuSurge, Medtronic) and the last 10 with a distal filter (FilterWire Ex, Boston Scientific). MDP was attempted if the culprit lesion was in a native coronary artery with a proximal reference diameter $\geq 3 \mathrm{~mm}$ and the angiographic appearance 5 min after administration of abciximab (standard intravenous bolus dose) remained highly suspicious for large residual thrombus. Patients in shock and with marked proximal vessel tortuosity were excluded. Pa-

\footnotetext{
${ }^{1}$ Section of Interventional Cardiology, Department of Cardiology, "A. De Gasperis," Niguarda Hospital, Milan, Italy

2Pathology Department, Niguarda Hospital, Milan, Italy

*Correspondence to: Dr. Pedro Silva Orrego, Via Bizzoni 5, 20125 Milano, Italy. E-mail: pedrosilva@tiscali.it
}

Received 27 December 2002; Revision accepted 9 June 2003

DOI $10.1002 / \mathrm{ccd} .10638$

Published online in Wiley InterScience (www.interscience.wiley.com). 
tients treated with fibrinolytic agents were also excluded. The choice of the interventional strategy was at the operator's discretion.

Distal embolization was defined as the migration of a filling defect or the distal occlusion of the target vessel or one of its branches at the end of the procedure or after positioning the MDP device. ECG recordings were analyzed by one observer blinded to the angiographic results. A 12 lead ECG was acquired at time of presentation and immediately after the intervention. The magnitude of ST segment elevation was measured 40 msec after the end of the QRS. ST segment score was calculated as the sum of ST segment elevation $>0.1 \mathrm{mV}$ for leads V1 through V6, aVL, and DI in anterior infarction, and for leads DII, DIII, aVF, V5, and V6 in inferior infarction. In case of true posterior infarction, reciprocal ST segment depressions $>0.1 \mathrm{mV}$ in V1 and V2 were also included. Persistent ST segment elevation $>50 \%$ in the postintervention ECG was considered to represent impaired reperfusion [16,17].

The protocol of the study was approved by the institutional review board of the Niguarda Hospital with written informed consent obtained from all patients before the procedure.

\section{PercuSurge Guardwire System}

The PercuSurge Guardwire system (Medtronic) is a device designed to prevent distal embolization during angioplasty and stenting of saphenous vein grafts [1315,18]. The device has no Food and Drug Administration (FDA) approval for use in native coronary arteries. The system has been previously described [15]. In brief, it consists of a guidewire (Guardwire) with a balloon incorporated distally for temporary occlusion and a monorail aspiration catheter (Export). The Guardwire is advanced as a conventional guidewire, positioning the occlusion balloon distal to the culprit lesion. The distal vessel can be seen with a small contrast injection through the guiding catheter to evaluate side branches and vessel diameter. When it is not possible to reach the lesion, a conventional wire can be used as a guiding rail. PCI is performed while the occlusion balloon is kept inflated distal to the lesion. After each balloon dilatation and/or stent deployment, debris can be aspirated through the Export catheter using a $20 \mathrm{cc}$ suction syringe before restoring distal flow by deflation of the balloon. For use in native coronary vessels, the smallest diameter $(3 \mathrm{~mm})$ distal occluding balloon was chosen in all cases.

\section{FilterWire Ex System}

This system has recently been used in SVG and native coronary arteries [19]. The device has no FDA approval as yet for use in native coronary arteries. The system consists of a core wire (diameter, $0.014^{\prime \prime}$ ) with an inte- grated filter basket at the distal end of the device to capture emboli liberated during the procedure. The basket is made of a nitinol ring and the filter is made of polyurethane (pore size, $80 \mu \mathrm{m}$ ). The ring is designed to fit into 3-5.5 mm diameter vessels. The device is inserted into the body with a rapid-exchange outer delivery sheath (3.9 Fr) that is used to hold the filter in the closed position. The sheath has a radiopaque marker at the distal tip for visualization under fluoroscopy. A guiding catheter lumen diameter of $6 \mathrm{Fr}$ is required (inner diameter, $\left.0.067^{\prime \prime}\right)$. Before insertion, it is recommended that a large dose of intracoronary nitroglycerin $(200-300 \mu \mathrm{g})$ be given to prevent vessel spasm. To negotiate the lesion, it is important to move the core wire and the outer sheath together, avoiding excessive rotation of the system to prevent loss of access and possible resistance to sheath withdrawal. If it is not possible to reach the lesion, a normal $0.014^{\prime \prime}$ wire can be used as a guiding rail. After crossing the lesion and reaching the distal site, a small contrast volume is injected to evaluate distal vessel diameter, curves, and side branches. Then the outer sheath is withdrawn back, allowing the filter deployment. At this point, it is very important to pull back the sheath, avoiding displacement of the core wire. The proximal marker of the filter should be positioned at least $1.5 \mathrm{~cm}$ distal to the lesion. After the procedure, the filter is retrieved in a closed position using the outer sheath. If it is difficult to cross the stented lesion with the sheath, a second type of sheath with an angulated tip can be used.

\section{Histopathology of Aspirated Material}

The material aspirated or retrieved with the filter was immediately fixed in $10 \%$ neutral buffered formalin and sent to pathology for analysis. After paraffin inclusion, 20 serial histologic sections (4 microns in thickness) were stained with hematoxylin and eosin. Results are expressed as mean \pm standard deviation.

\section{RESULTS}

From June 1999 to May 2002, we performed primary angioplasty with distal protection in 34 patients with acute myocardial infarction. In the first 24 cases, we used the PercuSurge Guardwire system, while the FilterWire Ex system was used in the last 10 cases.

Patients were not consecutive. MDP devices were not always available. These patients were treated with standard PCI and were not included in this registry. Selection was done according to protocol criteria (AMI within 12 hr not pretreated with fibrinolytics, with hemodynamic stability, in a native coronary artery with a vessel diameter $\geq 3 \mathrm{~mm}$ and without marked vessel tortuosity). When MDP devices were available, distal protection was attempted in all eligible patients. 
TABLE I. Clinical and Angiographic Characteristics

\begin{tabular}{lc}
\hline Age & $57 \pm 10$ years \\
Men & $85 \%$ \\
Admission systolic blood pressure & $117 \pm 27 \mathrm{~mm} \mathrm{Hg}$ \\
Admission heart rate & $72 \pm 19$ \\
Diabetes & $21 \%$ \\
Time, emergency room to wire & $81 \pm 71 \mathrm{~min}$ \\
Time, chest pain to wire & $280 \pm 180 \mathrm{~min}$ \\
CAD extension & \\
One-vessel & $65 \%$ \\
Two-vessel & $21 \%$ \\
Three-vessel & $14 \%$ \\
Culprit artery & $62 \%$ \\
RCA & $29 \%$ \\
LAD & $9 \%$ \\
LCx & \\
\hline
\end{tabular}

TABLE II. Procedural Data

\begin{tabular}{lc}
\hline Abciximab & $33 / 34(97 \%)$ \\
Vessel diameter & $3.62 \pm 0.6 \mathrm{~mm}$ \\
Procedural success & $32 / 34(94 \%)$ \\
Distal protection success & $28 / 32(88 \%)$ \\
PercuSurge & $19 / 22(86 \%)$ \\
Filter Wire & $9 / 10(90 \%)$ \\
\hline
\end{tabular}

The clinical and angiographic characteristics are summarized in Table I. Most patients were male, mean age was $57 \pm 10$ years, and $21 \%$ were diabetics. All patients were hemodynamically stable; only four patients upon admission had systolic blood pressure $<90 \mathrm{~mm} \mathrm{Hg}$. The mean ejection fraction was $44 \% \pm 11 \%$ in the 22 patients with the echocardiographic data available before or immediately after the procedure. The mean time elapsed from symptom onset to wire insertion was approximately $4 \mathrm{hr}$ and the mean time from admission to wire insertion was less than $90 \mathrm{~min}$. Most patients had single-vessel disease and the culprit artery was the right coronary artery (RCA) in 62\%, left anterior descendent (LAD) in $29 \%$, and left circumflex (LCx) in 9\% of patients.

Procedural data are shown in Table II. Abciximab was administered in standard dose, immediately before crossing the lesion with the wire in $97 \%$ of patients. The mean diameter of the culprit vessel was $3.62 \mathrm{~mm}$ (range, 3.01$5.06 \mathrm{~mm}$ ). The procedure was successful in 32 out of 34 cases $(94 \%)$. In two patients, the lesions were not crossable with standard wires and were treated medically. A distal protection device was successfully placed in 28 of the 32 patients (88\%), 19 out of $22(86 \%)$ with the PercuSurge system and in 9 out of 10 patients (90\%) with the FilterWire Ex system. The other four patients were treated with standard stenting after aspiration without protection of the distal vessel; one of these patients had distal embolization after stenting. To cross the lesion in the 28 patients with successful positioning of the distal protection device, a second wire was needed in 3 cases with FilterWire Ex (33\%) and in 10 with the PercuSurge system $(52 \%)$.

Table III shows the main results in the 28 patients effectively treated with distal protection. The baseline angiogram documented a mean diameter stenosis of $93 \%$ $\pm 5 \%$, a TIMI flow grade of $0-1$ in 25 patients $(89.2 \%)$, a TIMI 2 grade in 1 patient (3.6\%), and TIMI 3 flow grade in 2 patients (7.2\%). The culprit lesion was treated with a stent in 26 cases. In one patient, a good result without residual obstruction was obtained with aspiration only, while another patient required aspiration and balloon dilatation for a good result.

After the procedure, the residual stenosis was $2 \% \pm$ $10 \%$ and a TIMI 3 flow grade was observed in 24 patients (86\%): $84 \%$ in the group of patients treated with PercuSurge and $89 \%$ in the 8 patients treated with the FilterWire Ex. Worsening of the distal coronary flow, compared with the flow observed after the first balloon dilatation, was noted immediately after stenting in four patients $(21 \%)$ treated with PercuSurge (from TIMI 3 to TIMI 2) and in four patients (44\%) treated with the FilterWire Ex (two from TIMI 2 to TIMI 1 and two from TIMI 3 to TIMI 1). In all of these patients, after intracoronary administration of nitrates and adenosine, the final flow was normal.

The ST segment could not be interpreted in five patients because of a new bundle-branch block or the use of a ventricular pacemaker. Mean ST segment score before intervention was $12 \pm 6.5 \mathrm{~mm}$ and $4.8 \pm 5.3$ after the procedure. The ST segment elevation resolution $>50 \%$ was observed in $78 \%$ of patients $(73 \%$ with PercuSurge and $88 \%$ with the FilterWire Ex.

Angiographic evidence of distal embolization was observed in 4 patients: 2 out of $19(10 \%)$ in the PercuSurge group and 2 out of $9(22 \%)$ in the FilterWire group. In two cases (one PercuSurge and one FilterWire Ex), embolization was due to inadequate protection because of a bifurcation. In the other two cases, the embolization probably occurred while crossing the lesion. In two patients treated with the FilterWire Ex without angiographic evidence of embolization, a macroscopic thrombus was retrieved attached to the external part of the filter. In one case treated with the PercuSurge, thrombus was pulled back during aspiration to the proximal part of the artery, eventually being successfully removed with repeated aspirations. Distal coronary spasm was observed in two patients treated with the FilterWire Ex and was promptly reversed with intracoronary nitroglycerin. The MDP was difficult to retrieve in one case treated with FilterWire Ex, even with the second retrieval sheath, which did not cross the stented lesion. In this case, the filter was retrieved open and then we recrossed the lesion with a normal wire and redilated the stent without complications. 
TABLE III. Procedural and Clinical Results

\begin{tabular}{lccc}
\hline & & PercuSurge & FilterWire \\
\hline$\%$ stenosis pre & $98 \% \pm 5 \%$ & $93 \% \pm 5 \%$ & $92 \% \pm 6 \%$ \\
$\%$ stenosis post & $2 \% \pm 10 \%$ & $3 \% \pm 13 \%$ & $0 \pm 11 \%$ \\
TIMI 3 flow & & & \\
$\quad$ Basal & $2 / 28(7 \%)$ & $1 / 19(5 \%)$ & $1 / 9(11 \%)$ \\
$\quad$ Final & $24 / 28(86 \%)$ & $16 / 19(84 \%)$ & $8 / 9(89 \%)$ \\
ST segment score & $12.0 \pm 6.5 \mathrm{~mm}$ & $11.5 \pm 6.1 \mathrm{~mm}$ & $13.0 \pm 7.3 \mathrm{~mm}$ \\
$\quad$ Basal & $4.8 \pm 5.3 \mathrm{~mm}$ & $4.7 \pm 5.1 \mathrm{~mm}$ & $5.1 \pm 6.1 \mathrm{~mm}$ \\
$\quad$ Final & $18 / 23(78 \%)$ & $11 / 15(73 \%)$ & $7 / 8(88 \%)$ \\
ST reduction $>50 \%$ & $4 / 28(14 \%)$ & $2 / 19(10 \%)$ & $2 / 9(22 \%)$ \\
Distal embolization & $2 / 28(7 \%)$ & 0 & $2 / 9(22 \%)$ \\
Distal spasm & 0 & 0 & 0 \\
Urgent revascularization & 1 & 1 & 0 \\
Death & & & \\
\hline
\end{tabular}

One death due to cardiogenic shock occurred the day after the procedure in a patient treated with PercuSurge. No reinfarction or need for new urgent revascularization occurred during the in-hospital course.

In all cases treated with the PercuSurge system and in six out of nine patients with the FilterWire Ex, we retrieved visible particulate debris. Particle size ranged from 0.5 to $13 \mathrm{~mm}$ (Figs. 1 and 2). Pathologic study, available in 16 patients with a successful procedure, demonstrated recent nonorganized thrombus in 11 cases, fresh thrombus mixed with partially organized clot in 3 cases, and fresh plus partially organize clot with atherosclerotic plaque in 2 other cases.

\section{DISCUSSION}

Distal embolization is particularly frequent in certain conditions during percutaneous revascularization [20]. In fact, postprocedural infarction is higher after atherectomy and PCI in degenerated saphenous grafts [21,22]. It is also accepted that postprocedural enzymatic release correlates with long-term mortality [20,23]. PCI in saphenous grafts with distal protection using PercuSurge has a better TIMI flow and less non-Q-wave myocardial infarction compared with PCI without distal protection [14].

A major problem in the treatment of AMI is the potentiation of bulk emboli, promoted by either thrombolytic therapy or catheter-based reperfusion therapy [20]. In a posthoc analysis of primary angioplasty without stenting, Henriques et al. [12] document a $15 \%$ incidence of angiographically visible distal embolization, which is associated with more extensive myocardial damage and a poor prognosis. Abnormal coronary flow could be even worse after stenting [20,24]. The pivotal importance of microvascular obstruction during PCI is increasingly being recognized [20,25]. Use of improved antiplatelet therapy with anti-IIb/IIIa inhibitors has shown better outcome and coronary flow in patients with acute myocardial infarction treated with primary PCI
$[2,4,5]$. In patients with AMI, thrombus removal with the $\mathrm{X}$-Sizer catheter before standard PCI improves coronary epicardial flow and accelerates ST segment resolution compared with conventional PCI alone [16]. Entrapment devices in this setting is another approach to prevent embolic damage during percutaneous revascularization.

Mechanical protection from distal embolization in native coronary arteries during acute myocardial infarction has been reported in few patients $[15,19]$. In this study, we report our experience with two different systems for distal protection in native coronary arteries in 34 patients with acute myocardial infarction treated with primary PCI. Although this study was not done to compare the two systems of mechanical protection, the feasibility of the procedure was similar with both systems. Procedure failure in four cases (three PercuSurge and one FilterWire Ex) due to inability to negotiate the lesion was observed only at the beginning of our experience. Thereafter, probably because of a better selection of cases and the use of a second wire, we successfully positioned the distal protection device in the remaining cases. The majority of interventions was performed in the RCA. In fact, vessel morphology favors the RCA and limits the use of these devices in the LCx.

The $88 \%$ technical success rate of the procedure in this population, defined as positioning of the protection device distal to the lesion, is similar to the $90 \%$ to $96 \%$ reported by others using PercuSurge in SVG [14] or a porous filter in SVG or native coronary arteries [19]. Distal protection in the setting of acute myocardial infarction, however, has some differences compared with the mechanical protection reported in SVG $[14,19]$ or native coronary arteries in elective patients [19]. First of all, these devices were developed for SVG and carotid arteries, which are larger and less tortuous than native coronary arteries. Patients with acute myocardial infarction require rapid intervention, reducing the time needed to negotiate the lesion with this type of device. In addition, thrombus burden is always present, sometimes occluding the distal vessel, which carries the risk of embo- 


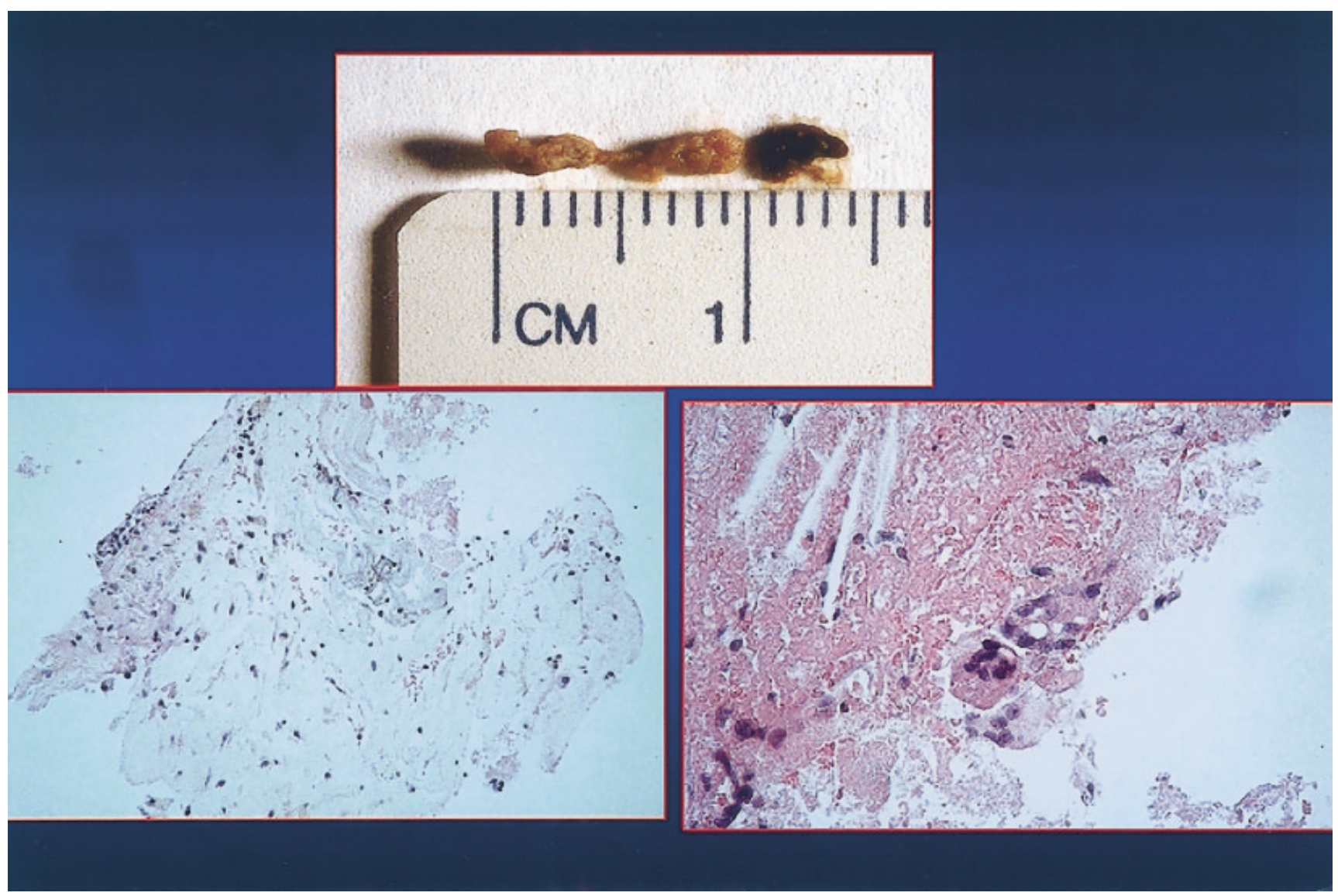

Fig. 1. Example of a thrombus aspirated from the Export catheter (top). Bottom left: The histologic section (hematoxylin-eosin stain, $40 \times$ ) shows at the left the lumen side superficially covered by flattened endothelial cells and, to the right, the plaque material composed of organizing/organized thrombus. Bottom right: The plaque material composed of cholesterol crystals circumscribed by multinucleated giant cells in an organized framework of fibrin. [Color figure can be viewed in the online issue, which is available at www.interscience.wiley.com.]

lization while crossing the thrombus or damaging the distal artery with the device itself.

We observed macroscopic distal embolization at the end of the procedure in $10 \%$ of cases treated with PercuSurge and $22 \%$ of patients treated with FilterWire Ex. Two other patients without angiographic evidence of embolization were found to have a small amount of thrombus on the external part of the filter. The $14 \%$ incidence of angiographically visible distal embolization in our patients is similar to that reported in patients treated with primary angioplasty without mechanical protection [12]. Small embolization can easily be missed on the angiogram. In fact, using contrast echocardiography, no tissue reperfusion has been observed in at least $25 \%$ of patients with reperfused AMI, even when brisk epicardial flow is present [26]. In this series of patients, embolic debris was retrieved in almost all patients, indicating that microembolization probably complicates all procedures of PCI in the setting of AMI.
The coronary TIMI flows at the end of the procedures were inferior to those reported in large trials with stent and abciximab without the use of distal protection devices [3]. We do not have an explanation for this observation, but we cannot exclude a detrimental effect of the device itself as suggested in the two cases with microvascular spasm resolved with adenosine.

Worsening of coronary flow has been reported after reperfusion in patients with acute myocardial infarction [27]. This deterioration seems worse after stenting compared with balloon dilatation alone [28]. It has been suggested that flow deterioration in this clinical situation is caused mainly by microembolization $[29,30]$. In this series of patients, we noted coronary flow worsening immediately after stenting in almost one-third of patients, including no reperfusion in four of them, which improved after intracoronary nitroglycerin or adenosine. Interestingly, flow deterioration immediately after stenting was worse and more frequent in patients treated with 


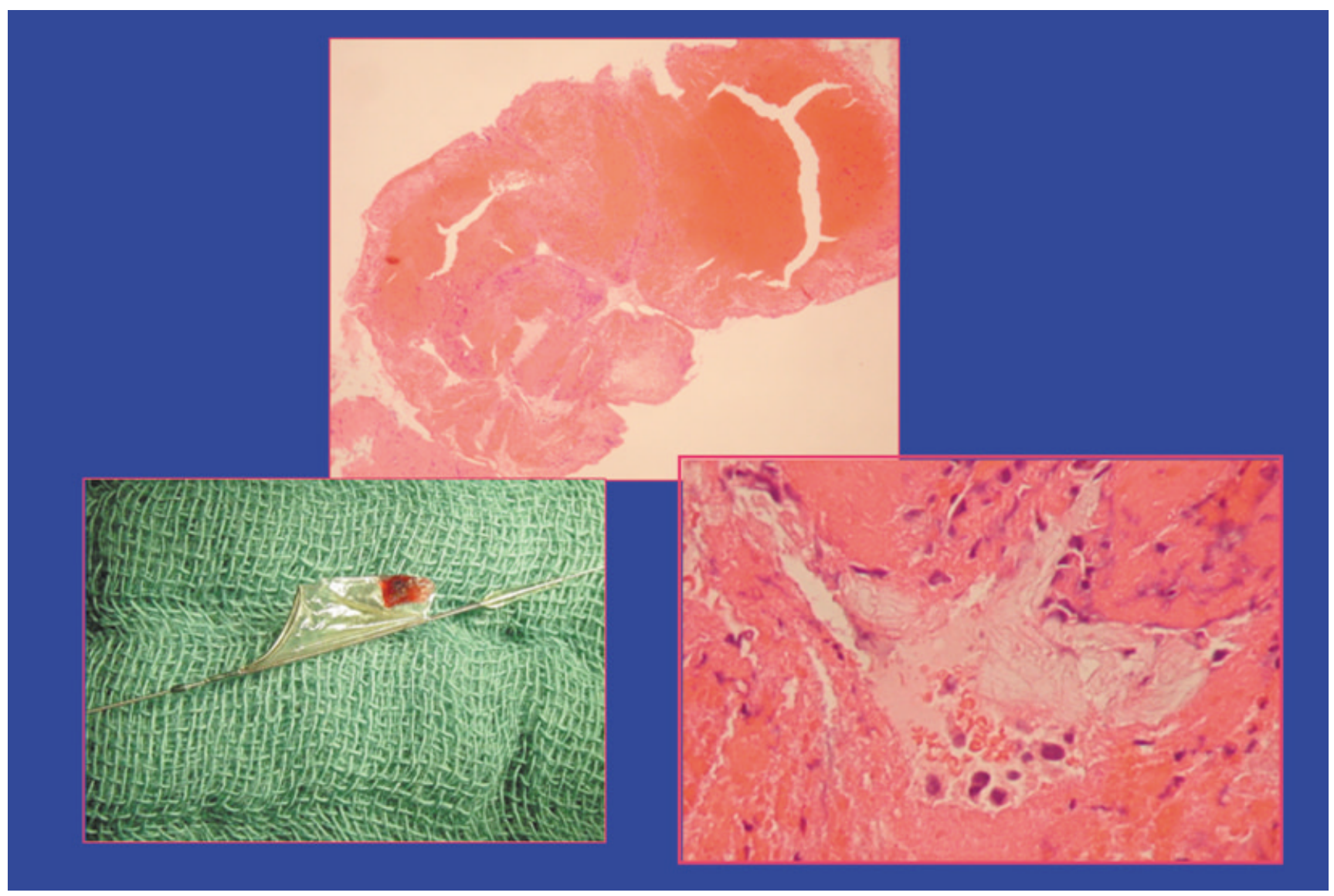

Fig. 2. An example of thrombus retrieved with the FilterWire EX (bottom left). The histologic section of the whole sample (hematoxylin-eosin stain, $40 \times$ ) shows the blood clot with plaque material (top). The close-up image (bottom right) shows hyaline substance infiltrated and organized by mononuclear cells. [Color figure can be viewed in the online issue, which is available at www.interscience.wiley.com.]

the filter protection system compared with the occlusion/ aspiration system, but the final TIMI flow was better in patients treated with the filter, suggesting a mechanism other than debris embolization.

Improved myocardial reperfusion, defined as ST segment resolution $>50 \%$ in the postprocedure ECG, was observed in approximately $80 \%$ of patients. This percentage of patients is clearly higher than the approximately $50 \%$ of patients with ST segment resolution treated with PCI without distal protection [16].

The principal limitations of this study are the relatively small number of patients, the nonrandomized nature of the study, and the lack of a control group. Comparing these results, however, with those reported in patients treated without distal protection, we observed a similar incidence of angiographic embolization [12] but improved myocardial reperfusion [16,31], suggesting that retrieving embolic debris with MDP may be beneficial in the treatment of AMI.
In conclusion, our study shows that mechanical distal protection in native coronary arteries during primary angioplasty can be done with minimal complications in selected patients. With these devices, the procedure is relatively easy and safe, embolic debris can be retrieved in almost all patients, and myocardial reperfusion is enhanced. Distal embolization still occurs in approximately $15 \%$ of patients. The development of new systems designed for native coronary arteries may improve results. Randomized trials are required to evaluate the clinical impact of distal protection in patients with acute myocardial infarction.

\section{REFERENCES}

1. Zahn R, Schiele R, Schneider S, et al. Primary angioplasty versus intravenous thrombolysis in acute myocardial infarction: can we define subgroups of patients benefiting most from primary angioplasty? results from the pooled data of the maximal individual 
therapy in acute myocardial infarction registry and the myocardial infarction registry. J Am Coll Cardiol 2001;37:1827-1835.

2. Schomig A, Kastrati A, Dirschinger J, et al. Coronary stenting plus platelet glycoprotein IIb/IIIa blockade compared with tissue plasminogen activator in acute myocardial infarction. $\mathrm{N}$ Engl J Med 2000;343:385-391.

3. Stone GW, Grines CL, Cox DA, et al. Comparison of angioplasty with stenting, with or without abciximab, in acute myocardial infarction (CADILLAC trial). N Eng J Med 2002;346:957-966.

4. Neumann FJ, Blasini R, Schmitt C, et al. A. Effect of glycoprotein IIb/IIIa receptor blockade on recovery of coronary flow and left ventricular function after placement of coronary-artery stents in acute myocardial infarction. Circulation 1998;98:2695-2701.

5. Schomig A, Kastrati A, Dirschinger J, et al. Coronary stenting plus platelet glycoprotein IIb/IIIa blockade compared with tissue plasminogen activator in acute myocardial infarction. N Engl J Med 2000;343:385-391.

6. Block PC, Elmer D, Fallon JT. Release of atherosclerotic debris after transluminal angioplasty. Circulation 1982;65:950-952.

7. MacDonald RG, Feldman RI, Conti CR, et al. Thromboembolic complications of coronary angioplasty. Am J Cardiol 1984;54: 916-917.

8. Colavita PG, Ideker RE, Reimer KA, et al. The spectrum of pathology associated with transluminal coronary angioplasty during acute myocardial infarction. J Am Coll Cardiol 1986;8:855860.

9. de Morais CF, Lopez EA, Checchi H, et al. Percutaneous transluminal coronary angioplasty: histopathologic analysis of nine necropsy cases. Wirchow Arch 1986;410:195-202.

10. Menke DM, Jordan MD, Aust CH, et al. Histologic evidence of distal coronary thromboembolism: a complication of acute proximal coronary artery thrombolysis therapy. Chest 1986;9:614616.

11. Saber RS, Edwards WD, Bailey KR, et al. Coronary embolization after balloon angioplasty or thrombolytic therapy: an autopsy study of 32 cases. J Am Coll Cardiol 1993;22:1283-1288.

12. Henriques JPS, Zijistra F, Ottervanger JP, de Boer MJ, van't Hof AWJ, Hoorntje JCA, Suryapranata H. Incidence and clinical significance of distal embolization during primary angioplasty for acute myocardial infarction. Eur Heart J 2002;23:1112-1117.

13. Carlino M, De Gregorio J, Di Mario M, et al. Prevention of distal embolization during saphenous vein graft lesion angioplasty: experience with a new temporary occlusion and aspiration system. Circulation 1999;99:3221-3223.

14. Baim DS, Wahr D, George B, et al., on behalf of the Saphenous Vein Graft Angioplasty Free of Emboli Randomized (SAFER) trial investigators. Randomized trial of a distal embolic protection device during percutaneous intervention of saphenous vein aortocoronary bypass grafts. Circulation 2002;105:285-290.

15. Belli G, Pezzano A, De Biase AM, et al. Adjunctive thrombus aspiration and mechanical protection from distal embolization in primary percutaneous intervention for acute myocardial infarction. Cathet Cardiovasc Intervent 2000;50:362-370.

16. Beran G, Lang I, Schreiber W, et al. Intracoronary thrombectomy with the X-Sizer catheter system improves epicardial flow and accelerates ST-segment resolution in patients with acute coronary syndrome: a prospective randomized study. Circulation 2002;105: 2355-2360.

17. Claeys MJ, Bosmans J, Venstra L, Jorens P, De Raedt H, Vrints CJ. Determinants and prognostic implications of persistent STsegment elevation after primary angioplasty for acute myocardial infarction. Circulation 1999;99:1972-1977.

18. Grube E, Schofer J, Webb J, et al. Evaluation of a balloon occlusion and aspiration system for protection from distal embolization during stenting saphenous vein grafts: the Saphenous Vein Graft Angioplasty free of Emboli (SAFE) trial study group. Am J Cardiol 2002;89:941-945.

19. Grube E, Gerkens U, Yeung A, et al. Prevention of distal embolization during coronary angioplasty in saphenous vein grafts and native vessels using porous filter protection. Circulation 2001;104: 2436-2441.

20. Topol EJ, Yadav JS. Recognition of the importance of embolization in atherosclerotic vascular disease. Circulation 2000;101: $570-580$.

21. Topol EJ, Leya F, Pinkerton CA, et al., for the CAVEAT study group. A comparison of directional atherectomy with coronary angioplasty in patients with coronary artery disease. N Engl J Med 1993;329:221-227.

22. Holmes DR, Topol EJ, Califf RM, et al. A multicenter, randomized trial of coronary angioplasty versus directional atherectomy for patients with saphenous vein bypass graft lesions. Circulation 1995;91:1966-1974.

23. Saucedo JF, Mehran R, Dangas G, et al. Long-term clinical events following creatine kinase myocardial band isoenzyme elevation after successful coronary stenting. J Am Coll Cardiol 2000;35; $1134-1141$

24. Kern MJ, Puri S, Bach RG, et al. Abnormal coronary flow velocity reserve after coronary artery stenting in patients: role of relative coronary reserve to assess potential mechanisms. Circulation 1999;100:2491-2498.

25. Matthew TR, Ohman EM, Maas A, et al. Shifting the open-artery hypothesis downstream: the quest for optimal reperfusion. J Am Coll Cardiol 2001;37:9-18.

26. Ito $\mathrm{H}$, Maruyama A, Iwakura $\mathrm{K}$, et al. Clinical implications of the no-reflow phenomenon: a predictor of comlications anf left ventricular remodeling in reperfused anterior wall myocardial infarction. Circulation 1996;93:223-228.

27. IwakuraK, Ito H, Nishikawa L, et al. Early temporal changes in coronary flow velocity patterns in patients with acute myocardial infarction demonstrating the "no-reflow" phenomenon. Am J Cardiol 1999;84:415-419.

28. Grines CL, Cox DA, Stone GW, et al. Coronary angioplasty with or without stent implantation for acute myocardial infarction. N Engl J Med 1999;341:1949-1956.

29. Rezkalla S, Kloner RA. No-reflow phenomenon. Circulation 2002;105:656-662.

30. Erbel R, Heusch G. Coronary microembolization. J Am Coll Cardiol 2000;36:22-24.

31. Poli A, Fetiveau R, Vandoni $P$, et al. Integrated analysis of myocardial blush and st-segment elevation recovery after successful primary angioplasty. real-time grading of microvascular reperfusion and prediction of early and late recovery of left ventricular function. Circulation 2002;106:313-318. 